\title{
An experimental and numerical study on the mechanical properties of carbon nanotube-latex thin films
}

Authors: Long Wang ${ }^{\mathrm{a}, 1}$

Kenneth J. Loh ${ }^{\mathrm{a}, 2, *}$

Lucas Brely ${ }^{\mathrm{b}, 3}$

Federico Bosia ${ }^{\mathrm{b}, 4}$

Nicola M. Pugno c,d,e,5,*

${ }^{a}$ Department of Structural Engineering, University of California, San Diego, 9500 Gilman Drive, Mail Code 0085, La Jolla, CA, 92093-0085, USA

b Department of Physics and "Nanostructured Interfaces and Surfaces" Centre, Università di Torino, Italy

${ }^{c}$ Laboratory of Bio-Inspired \& Graphene Nanomechanics, Department of Civil, Environmental and Mechanical Engineering, Università di Trento, Italy

${ }^{\mathrm{d}}$ Center for Materials and Microsystems, Fondazione Bruno Kessler (Trento), Italy

${ }^{\mathrm{e}}$ School of Engineering and Materials Science, Queen Mary University of London, UK

1 e-mail: lowang@ucdavis.edu

2 e-mail: kenloh@ucsd.edu

3 e-mail: lucasleo.brely@unito.it

4 e-mail: federico.bosia@unito.it

5 e-mail: Nicola.Pugno@unitn.it

*Co-corresponding author 


\begin{abstract}
Multi-walled carbon nanotube (MWNT)-latex composite thin films of different MWNT concentrations were fabricated by spraying. Post-fabrication thermal annealing was then conducted on samples sets of different MWNT concentrations, and their microstructure, morphology, and mechanical properties were compared to non-annealed sample sets. The incorporation of up to $3 \mathrm{wt} \%$ MWNTs enhanced the mechanical properties of these nanocomposites. In addition, annealing altered the microstructure and morphology of the latex matrix, which enhanced the interactions between MWNTs and the polymer to significantly increasing their ultimate failure strain and tensile strength. Furthermore, the reinforcing effects of MWNTs on the polymer matrix were investigated using numerical simulations. Stress concentrations were found to initiate at MWNT ends, thus giving rise to yielding fronts that tend to coalesce and propagate across the entire film. The enhancement of the mechanical properties of MWNT-latex nanocomposites make them more suitable for field application as multifunctional coatings or sensors.
\end{abstract}

Keywords: annealing; carbon nanotube; mechanical property; nanocomposite; numerical simulation

\title{
1. Introduction
}

Carbon nanotubes (CNT) have received significant attention and have been studied extensively since the work by Iijima [1]. Early research has focused on characterizing individual CNTs' intrinsic properties, which include their unique aspect ratios, low density, mechanical [2-4], and electrical [5] properties. In addition, they have been shown to be piezoresistive [6-8] and sensitive to thermal effects [9], among others, which make them prime candidates for multifunctional materials. In fact, their unique properties have been leveraged for developing various CNT-based sensing devices [8, 10]. However, the application of individual CNTs can be challenging due to their small dimension, especially when it comes to large-scale civil, aerospace, and marine structural applications.

On the other hand, CNTs serve as ideal reinforcing materials for engineered composites due to their unique mechanical properties [11-13]. After CNTs are incorporated within materials such as polymer matrices, they can be more readily used as macro-scale nanocomposites for large-scale applications. Examples include using vacuum filtration (for buckypapers) [14], layer-by-layer (LbL) deposition [15], and electrospinning [16], to name a few. Although these methods could successfully fabricate piezoresistive CNT-based thin films, they suffer from limitations, including the use of complicated or time-consuming fabrication procedures, low productivity, and size constraints. In contrast, spray-coating has been investigated as a viable alternative, since it is simple, efficient, low cost, and uses readily available raw materials (e.g., latex) [17, 18]. A previous study by Wang and Loh [19] showed that spray-coated, post-fabrication, thermally annealed MWNT-latex thin 
films exhibited improved electromechanical properties; nominal electrical resistance drifts were eliminated or mitigated, which make them more suitable for use as strain sensors for structural health monitoring applications.

The objective of this study was to characterize how nanotube concentrations and postfabrication thermal annealing affected the bulk mechanical properties of the aforementioned spray-coated MWNT-latex thin films. Monotonic uniaxial tensile tests were conducted so as to not only evaluate the mechanical properties of MWNT-latex composites but to also characterize the effects of MWNT concentrations and annealing on bulk film mechanical properties. The differences observed in mechanical parameters among different sample sets were then correlated with their microstructures. In addition, numerical simulations based on a spring model approach ([20,21]) were conducted to study the reinforcing and stiffening effects of MWNTs dispersed in a similar polymer matrix. Stress distributions in the films were mapped, and the effect of MWNT distributions on the onset of yielding effects and damage propagation was highlighted.

\section{Experimental Details}

\subsection{Materials}

The MWNTs used in this study were purchased from SouthWest NanoTechnologies, whose outer diameter and length were 6-9 $\mathrm{nm}$ and $5 \mu \mathrm{m}$, respectively; their purity exceeded $95 \%$. Poly(sodium 4-styrenesulfonate) (PSS) $\left(M_{w} \approx 1 \mathrm{M}\right)$ and N-methyl-2-pyrrolindinone (NMP) were from Sigma-Aldrich. The latex solution was from Kynar Aquatec. Other disposable laboratory supplies were from Fisher Scientific. All materials were used as acquired without further purification.

\subsection{Thin film preparation}

MWNT-latex nanocomposite films were spray-fabricated using an airbrush, following the procedure described in [19]. In short, the fabrication procedure involved two major steps. First, to prepare the spray-able MWNT-latex inks, a mixture of MWNTs, 2 wt\% PSS aqueous solution, and trace amounts of NMP was subjected to ultrasonication. Then, appropriate amounts of latex solution and $18 \mathrm{M} \Omega$ deionized water were mixed with the sonicated solution. The amount of MWNTs were determined based on the fact that the final sprayed films would possess concentrations of 1, 2, and $3 \mathrm{wt} \%$ MWNTs.

Second, the MWNT-latex inks were manually sprayed onto $25 \times 75 \mathrm{~mm}^{2}$ glass microscope slides using a Paasche airbrush. The films were air dried in the fume hood for at least $3 \mathrm{~h}$, after which freestanding films were obtained by releasing them from the substrates. In addition, 0 wt $\%$ thin films were also fabricated as a control set, following the same procedure, except that no MWNTs were added to the PSS-NMP solution. It should be mentioned that several sets of 1,2, and $3 \mathrm{wt} \%$ annealed MWNT-latex thin films were also prepared. For these sample sets, post-fabrication thermal annealing was conducted using a 
StableTemp Model 282A vacuum oven. These freestanding nanocomposite films were subjected to a temperature of $80^{\circ} \mathrm{C}$ for $12 \mathrm{~h}$, followed by $150{ }^{\circ} \mathrm{C}$ for $3 \mathrm{~h}$ in vacuum.

\subsection{Thin film characterization}

The effects of MWNT concentrations and thermal annealing on the microstructure of the MWNT-latex films were measured by X-ray diffraction (XRD, ScintagXRD). Atomic force microscopy (AFM, Asylum MFP-3D AFM) was utilized for analyzing the films' surface topography. MWNT dispersion, as well as changes in film morphology after annealing, were investigated with scanning electron microscopy (SEM, Philips/FEI XL30 SFEG SEM).

The mechanical properties of MWNT-latex nanocomposites were characterized by conducting monotonic uniaxial tensile tests on freestanding thin films. Here, non-annealed $0,1,2$, and $3 \mathrm{wt} \%$ and annealed 1, 2, and $3 \mathrm{wt} \%$ thin films were investigated. The freestanding films were cut into smaller specimens of $5 \times 55 \mathrm{~mm}^{2}$ and then mounted in a Test Resources 150R load frame for testing. Tensile load was applied using a load rate of $1 \%-\min ^{-1}$ until film failure. During testing, the displacement of the crosshead was measured using an MTI laser displacement transducer connected to an Agilent 34401A digital multimeter (DMM), which was recorded and time-synchronized with load measurements from the load frame using a customized LabVIEW program. It should be mentioned that no detectable relative slippage between the thin film and the crossheads occurred during the tests owing to the use of an appropriate set of rubber-coated grips.

\subsection{Numerical simulations model}

The numerical model employed for simulating the mechanical behavior of MWNT-latex films was adapted from a recently developed spring model approach. In additional, a nonlocal lattice formulation was adopted to avoid preferential crack propagation in specific directions due to the regular lattice configuration, therefore providing a more realistic model $[22,23]$. As schematically shown in Fig. 1a, a representative material portion was discretized into a set of nodes, each linked to its neighbors through nonlinear "springs". The constitutive elasto-plastic behavior is shown schematically in Fig. 1b. The non-linearity was introduced in the code through a forward Eulerian incremental procedure ([24]) using a small strain increment $(0.1 \%)$ at each simulation step. Elastic constants, yield point, and plastic behavior of the latex matrix were obtained from experimental stress-strain curves and average mechanical parameters obtained in this study.

Randomly oriented line inclusions, discretized into a set of equidistant nodes, were introduced into the model to simulate the behavior of the MWNT reinforcements (Fig. 1c). The approach was similar to that adopted in [25], where the reinforcements were treated as 1D "anticracks" in a 2D soft matrix. In our approach, it was assumed that no relative displacements are possible between the reinforcement representative nodes, due to the largely superior MWNT stiffness with respect to the matrix. Inclusion nodes are connected

to matrix neighboring nodes with "interface" bonds having the same properties as matrix 
bonds to simulate a perfect interface. A uniaxial tensile test in displacement control was then simulated on a model sample (Fig. 1d) to obtain the stress-strain behavior of the nanocomposite. Periodic boundary conditions were applied on the lateral boundaries of the sample. Due to the geometry of the problem, a plane stress approximation was adopted.

\section{Results and Discussion}

\subsection{Microstructure and morphology characterization}

The effects of incorporating MWNTs in latex, as well as annealing, on the microstructure of these nanocomposites were measured using XRD, and representative results are shown in Fig. 2. First, non-annealed $0 \mathrm{wt} \%$ thin films (i.e., latex-PSS) showed a broad diffraction peak at $2 \theta=16.5^{\circ}$, which indicates that their microstructure was mainly amorphous. On the other hand, pristine MWNTs exhibited two dominant peaks in their diffraction pattern, which were located at $2 \theta=26^{\circ}$ and $43^{\circ}$, corresponding to (002) and (100) Bragg reflections, respectively [26-28]. The (002) peak indicates the inter-shell spacing of the MWNTs is $3.4 \AA$, while the (100) one is due to stacking of nanotubes within MWNTs. It should be noted that other small peaks captured by XRD might due to MWNT impurities. Second, the XRD results of non-annealed films that incorporated MWNTs, which are shown in Fig. 2a (i-iv), possess two peaks at $2 \theta=16.5^{\circ}$ and $26^{\circ}$ and are contributed by the polymer matrices and MWNTs, respectively. This means that the incorporation of MWNTs in the polymer matrix did not generate any formation of secondary phases, and MWNTs were randomly oriented. The diffraction peak intensities of MWNTs were also more pronounced in nanocomposites with higher MWNT concentrations. Furthermore, it was found that annealed MWNT-latex films did not show any significant differences than their nonannealed counterparts (Fig. 2b), thereby indicating that post-fabrication thermal treatment did not introduce additional order in the microstructure of these nanocomposites.

The surface topography of MWNT-latex films was investigated using AFM. For the nonannealed films, Figs. 3a (a $0 \mathrm{wt} \%$ film) and 3c (a $1 \mathrm{wt} \%$ film) highlight spherical latex particles with an average diameter of $\sim 100 \mathrm{~nm}$, forming a closely overlapped and compact matrix. In Fig. 3c, the MWNTs can be observed on the surface of the films and are separately distributed in the latex matrix. In contrast, spherical latex particles in annealed films were hardly discernable, regardless of whether MWNTs were incorporated or not (Figs. $3 \mathrm{~b}$ and $3 \mathrm{~d}$ ). This result indicates that post-fabrication annealing changed the configuration of latex particles and altered the polymer microstructure. Moreover, one can also observe from the amplitude scale bar in Fig. 3 that the films' surfaces became smoother after annealing.

MWNT dispersion, changes in polymer matrix configurations, and fracture surfaces of MWNT-latex thin films were also characterized by SEM. Several findings can be summarized by comparing the SEM images of non-annealed and annealed $1 \mathrm{wt} \%$ thin films as shown in Fig. 4. First, MWNTs were homogeneously distributed and randomly oriented in the polymer matrix, rather than aggregated into bundles. Second, the spherical latex 
particles that were present in the polymer matrix in the non-annealed films (Figs. 4a and 4c) disappeared after annealing (Figs. $4 \mathrm{~b}$ and $4 \mathrm{~d}$ ). Moreover, there were many voids (larger than $300 \mathrm{~nm}$ ) in non-annealed thin films, which may be due to the slow degassing of the MWNT-latex ink after their deposition, especially when considering the viscosity of the ink solution and trapped air bubbles after spraying. In comparison, the polymer matrices of annealed specimens were more intact, and MWNTs were better integrated with latex.

Furthermore, the fracture surfaces of non-annealed and annealed MWNT-latex thin films were also investigated using SEM. High magnification SEM images of fractured surfaces of representative non-annealed and annealed films are shown in Figs. $4 \mathrm{c}$ and $4 \mathrm{~d}$, respectively. For both cases, one can observe nanotubes being pulled out of the polymer matrix. However, the diameter of the pulled out fibers for the annealed films (Fig. 4d) were larger than $\sim 150 \mathrm{~nm}$. This suggests that MWNTs were wrapped by polymers, which indicates that the MWNT-polymer interaction forces might be greater in the annealed versus non-annealed ones. In addition, since the melting point of latex generally falls between 120 and $176{ }^{\circ} \mathrm{C}$, depending on its constituents, annealing could soften the latex matrix. The softened latex was able to viscously flow around MWNTs and fill voids while curing defects in the polymer matrix. As a result, annealed films should provide greater resistance to nanotube pull-out and enhanced fracture properties due to stronger MWNTpolymer interactions. Finally, delamination occurred in selected specimens for both nonannealed and annealed films (Figs. $4 \mathrm{a}$ and $4 \mathrm{~b}$ ). Since the films were air-dried, the top layers potentially dried faster than the bottom, which affected film integrity in the thickness direction.

\subsection{Mechanical characterization}

Monotonic uniaxial tensile tests were conducted as mentioned in Section 2.3. Fig. 5 shows representative stress-strain curves of non-annealed $0,1,2$, and $3 \mathrm{wt} \%$ nanocomposite films, in which an initial linear-elastic, followed by a yielding and hardening region, can be identified. The stress-strain profiles of annealed films possessed the same pattern, and thus, are not shown here. To define the yield point, two linear least-squares regression lines were fitted to characterize the initial elastic region and the hardening region. The intersection of the two fitted lines were then assumed to be the yield point, as shown in the inset of Fig. 5. Then, the elastic modulus of the nanocomposites was determined using the slope of the linear best-fit line corresponding to the initial elastic region in the stress-strain curve.

Figs. 6 to 8 summarize the different trends in the mechanical properties of non-annealed and annealed MWNT-latex thin films of different MWNT concentrations. It is obvious that, for both non-annealed and annealed thin films, the dispersion of MWNTs in the polymer matrix should and did improve their tensile strengths and elastic moduli, as shown in Figs. 6 and 7a. MWNTs would resist crack formation and bridge micro-cracks in the polymer matrix when the nanocomposites were stretched, thereby improving their strength [29]. This was evident from the SEM images of fracture surfaces discussed in Section 3.1 (see Fig. 4). At the same time, due to the MWNT-latex interfacial forces, the nanocomposites required larger external forces to overcome the greater inter-molecular friction to produce 
the same deformation as $0 \mathrm{wt} \%$ ones, thereby increasing the stiffness of the films. As MWNT concentrations increased, the strengthening and stiffening effects became more prominent, which is consistent with the trend observed in the XRD peaks associated with MWNTs (see Fig. 2). Besides, Fig. 7a shows an approximately linear increasing trend in tensile strength as MWNT concentration increased, again suggesting that at least up to 3 wt\% MWNTs could be effectively dispersed in the polymer matrix using the sprayfabrication technique employed in this study.

Furthermore, as compared with non-annealed films of the same MWNT concentration, annealed ones exhibited higher tensile strengths but lower elastic moduli, which indicates that thermal treatment could strengthen the thin films while soften the material. Since annealing cured voids in the matrix, MWNTs became better incorporated within the polymer matrix according to the SEM images (see Fig. 4). Annealed films featured higher integrity (i.e., fewer defects), which provided them with higher tensile strengths than their non-annealed counterparts. However, the reduced stiffness could be mainly caused by possible changes in the configuration of the polymer matrix after annealing.

On the other hand, Fig. $7 \mathrm{~b}$ shows that the ultimate failure strain of non-annealed films decreased as more MWNTs were added. The drop was significant between $0 \mathrm{wt} \%$ to 1 wt $\%$, and tended to level off beyond that, indicating that thin films with more dispersed MWNTs had less flexibility. This occurred because the MWNT network acts like crosslinks in the polymer matrix, which constrains polymer chains from moving under tensile stress [30]. However, annealed thin films exhibited significantly higher ultimate failure strains than non-annealed ones, especially for the $1 \mathrm{wt} \%$ set, whose average strain at failure increased up to $\sim 300 \%$. Therefore, thermal treatment could enhance the flexibility of MWNT-latex thin films, and the improvement was more remarkable on films of lower MWNT concentrations. The effects of annealing on the flexibility could be attributed to the change in the polymer matrix configuration (see Fig. 3). Besides, such enhancements would be more significant in films that possessed higher polymer content, which explains why decreasing MWNT concentration led to an increase in ultimate failure strain after annealing.

Similarly, Figs. 8a and 8b show that the derived yield stress increased as MWNT concentrations increased while yield strain decreased, which was true for both nonannealed and annealed thin films. In addition, thermal treatment consistently increased both the yield stress and yield strain of the films with regard to MWNT concentrations. The aforementioned effects of MWNT concentrations and annealing may also apply here.

\subsection{Numerical simulations characterization}

Simulations provide numerical predictions for the overall mechanical behavior of the experimentally measured specimens characterized by different MWNT concentrations. The stress-strain curves are presented in Fig. 9. Experimental data for the unreinforced matrix were schematically modeled as an initial linear-elastic phase up to the yield point at $3.1 \%$ strain, and a subsequent plastic phase with a 60 -fold reduced slope up to failure at a $7 \%$ 
ultimate strain. These matrix properties were used in simulations for the reinforced composite. The stress-strain curves obtained for increasing reinforcement weight fractions have a similar behavior to that observed experimentally. Increasing MWNT concentrations give rise to a stiffer material with yielding at smaller strain values (i.e., $1.4 \%$ for $1 \mathrm{wt} \%, 1.2$ $\%$ for $2 \mathrm{wt} \%$, and $1 \%$ for $3 \mathrm{wt} \%$ ). This is due to increased stress concentrations in matrix sub-regions where additional load is transferred via the reinforcements. As expected, increasing MWNT concentrations also give rise to greater strength (i.e., 8.4 MPa for 1 $\mathrm{wt} \%, 13.4 \mathrm{MPa}$ for $2 \mathrm{wt} \%$, and $17.9 \mathrm{MPa}$ for $3 \mathrm{wt} \%$ ). It can be seen that stresses are underestimated with respect to experimental values. A probable reason is due to the numerical results being conservative, where higher stress concentrations were imposed by considering CNTs as rigid.

Simulations also allow for mapping stress distributions within the deforming nanocomposite and for highlighting their evolution during applied increasing loading levels. An example is shown in Fig. 10 for a material portion of approximately $9 \times 12 \mu \mathrm{m}^{2}$. The reinforcements embedded in the softer matrix take up the strains applied on the surrounding zone, and the images show that line inclusions, due to their high aspect ratio, lead to stress concentrations mainly located at their tips. Stress concentrations are initially distributed randomly inside the matrix and act as "seeds" for the onset of yielding, leading to the propagation of plastic fronts. The evolution of plastification is highlighted in Figs. $10 \mathrm{~b}$ and 10c, where plastic zones tend to merge and spread, unless shielded by other MWNTs. This was further highlighted by analyzing the strain distributions in the polymer matrix at different applied strain levels, $\varepsilon$, as shown in Fig. 11. Relative frequency histograms of the strains were provided for the three MWNT concentrations considered in this study, namely, $1 \mathrm{wt} \%$ (Fig. 11a), $2 \mathrm{wt} \%$ (Fig. 11b), and $3 \mathrm{wt} \%$ (Fig. 11c). In each case, a widening of the distributions is observed, especially at high applied strain levels that were well above the yield strain $(8 \%)$. This indicates that maximum strains most likely first concentrated at MWNT crack ends, where the initial large strain usually occurred. Then, the nanocomposite would evolve to a highly-damaged condition, in which most parts of the polymer matrix would be in a yielded state. Also, it should be noted that both compressive and tensile strains would occur during this process, due to the complex nature of damage propagation in heterogeneous materials. The observed trend was enhanced as MWNT concentrations increased in the specimens.

\section{Conclusions}

In this study, MWNT-latex nanocomposite thin films were fabricated by spraying, and up to $3 \mathrm{wt} \%$ MWNTs were homogeneously distributed in the latex polymer matrix. The incorporation of MWNTs in the polymer matrix tended to strengthen and stiffen the nanocomposite, and the effects became more significant as MWNT concentrations increased. This indicates that MWNTs acted effectively as reinforcing fillers in the thin films. In addition, to improve the mechanical properties of MWNT-latex thin films, postfabrication thermal annealing was carried out. It was found that annealing could enhance flexibility and tensile strength. Such enhancement was mainly caused by changes in the 
latex matrix configuration after annealing, which generated stronger interfacial forces between MWNTs and the polymer matrix and formed a more homogeneous microstructure. Based on the numerical simulation results, randomly distributed MWNTs in the polymer matrix could effectively strengthen and stiffen the nanocomposite, which agreed with experimental results. Numerical simulations also showed that, upon loading, stresses tended to concentrate at MWNT ends, subsequently leading to local yielding effects that propagated across the entire thin film, thereby explaining the observed elasto-plastic constitutive behavior.

Overall, this work presents a simple, scalable, effective, and low cost fabrication technique suitable for creating MWNT-latex thin films characterized by favorable mechanical and electromechanical properties. Future studies will focus on further developing the spray fabrication technique for two main goals. One is to fabricate MWNT-latex thin films with a broader range of MWNT concentrations so as to investigate whether the findings derived from this study continue to apply. The second is to employ spray coating for fabricating other types of multifunctional nanocomposites.

\section{Acknowledgements}

This research was supported by the U.S. National Science Foundation (NSF) under grant number CMMI-CAREER 1253564 and supplement CMMI-CAREER 1542532. In addition, NMP acknowledges support by the European Research Council (ERC StG Ideas 2011 BIHSNAM no. 279985, ERC PoC 2013-1 REPLICA2 no. 619448, ERC PoC 2013-2 KNOTOUGH no. 632277), by the European Commission under the Graphene Flagship (WP10 'Nanocomposites', no. 604391) and by the Provincia Autonoma di Trento ('Graphene nanocomposites', no. S116/2012-242637 and reg. delib. no. 2266). LB and FB are supported by BIHSNAM.

\section{References}

[1] S. Iijima. Helical microtubules of graphitic carbon. Nature. 354 (6348) (1991), pp. 5658.

[2] J.-P. Salvetat, G. A. D. Briggs, J.-M. Bonard, R. R. Bacsa, A. J. Kulik, T. Stöckli, N. A. Burnham, L. Forró. Elastic and shear moduli of single-walled carbon nanotube ropes. Physical review letters. 82 (5) (1999), pp. 944-947.

[3] M.-F. Yu, B. S. Files, S. Arepalli, R. S. Ruoff. Tensile loading of ropes of single wall carbon nanotubes and their mechanical properties. Physical review letters. 84 (24) (2000), pp. 5552-5555.

[4] D. Walters, L. Ericson, M. Casavant, J. Liu, D. Colbert, K. Smith, R. Smalley. Elastic strain of freely suspended single-wall carbon nanotube ropes. Applied Physics Letters. 74 (25) (1999), pp. 3803-3805.

[5] T. Ebbesen, H. Lezec, H. Hiura, J. Bennett, H. Ghaemi, T. Thio. Electrical conductivity of individual carbon nanotubes. Nature. 382 (1996), pp. 54-56. 
[6] T. W. Tombler, C. Zhou, L. Alexseyev, J. Kong, H. Dai, L. Liu, C. Jayanthi, M. Tang, S.-Y. Wu. Reversible electromechanical characteristics of carbon nanotubes under local-probe manipulation. Nature. 405 (6788) (2000), pp. 769-772.

[7] J. Cao, Q. Wang, H. Dai. Electromechanical properties of metallic, quasimetallic, and semiconducting carbon nanotubes under stretching. Physical review letters. 90 (15) (2003), pp. 157601-157604.

[8] R. J. Grow, Q. Wang, J. Cao, D. Wang, H. Dai. Piezoresistance of carbon nanotubes on deformable thin-film membranes. Applied Physics Letters. 86 (9) (2005), pp. 093104-093106.

[9] M. Fujii, X. Zhang, H. Xie, H. Ago, K. Takahashi, T. Ikuta, H. Abe, T. Shimizu. Measuring the thermal conductivity of a single carbon nanotube. Physical review letters. 95 (6) (2005), pp. 065502-065505.

[10] S. J. Tans, A. R. Verschueren, C. Dekker. Room-temperature transistor based on a single carbon nanotube. Nature. 393 (6680) (1998), pp. 49-52.

[11] J. N. Coleman, U. Khan, W. J. Blau, Y. K. Gun'ko. Small but strong: a review of the mechanical properties of carbon nanotube-polymer composites. Carbon. 44 (9) (2006), pp. 1624-1652.

[12] A. K.-T. Lau, D. Hui. The revolutionary creation of new advanced materials-carbon nanotube composites. Composites Part B: Engineering. 33 (4) (2002), pp. 263-277.

[13] J. N. Coleman, U. Khan, Y. K. Gun'ko. Mechanical reinforcement of polymers using carbon nanotubes. Advanced materials. 18 (6) (2006), pp. 689-706.

[14] P. Dharap, Z. Li, S. Nagarajaiah, E. Barrera. Nanotube film based on single-wall carbon nanotubes for strain sensing. Nanotechnology. 15 (3) (2004), pp. 379-382.

[15] K. J. Loh, J. Kim, J. P. Lynch, N. W. S. Kam, N. A. Kotov. Multifunctional layer-bylayer carbon nanotube-polyelectrolyte thin films for strain and corrosion sensing. Smart Materials and Structures. 16 (2) (2007), pp. 429-438.

[16] F. Ko, Y. Gogotsi, A. Ali, N. Naguib, H. Ye, G. Yang, C. Li, P. Willis. Electrospinning of continuous carbon nanotube-filled nanofiber yarns. Advanced materials. 15 (14) (2003), pp. 1161-1165.

[17] P.-C. Ma, N. A. Siddiqui, G. Marom, J.-K. Kim. Dispersion and functionalization of carbon nanotubes for polymer-based nanocomposites: a review. Composites Part A: Applied Science and Manufacturing. 41 (10) (2010), pp. 1345-1367.

[18] N. Grossiord, J. Loos, O. Regev, C. E. Koning. Toolbox for dispersing carbon nanotubes into polymers to get conductive nanocomposites. Chemistry of materials. 18 (5) (2006), pp. 1089-1099.

[19] L. Wang, K. J. Loh. Spray-coated carbon nanotube-latex strain sensors. Science Letters Journal. 5 (234) (2016).

[20] L. Brely, F. Bosia, N. M. Pugno. A hierarchical lattice spring model to simulate the mechanics of 2-D materials-based composites. Frontiers in Materials. 2 (2015),

[21] S. Panzavolta, B. Bracci, C. Gualandi, M. L. Focarete, E. Treossi, K. KouroupisAgalou, K. Rubini, F. Bosia, L. Brely, N. M. Pugno. Structural reinforcement and failure analysis in composite nanofibers of graphene oxide and gelatin. Carbon. 78 (2014), pp. 566-577.

[22] P. D. Beale, D. J. Srolovitz. Elastic fracture in random materials. Physical Review B. 37 (10) (1988), 5500. 
[23] H. Chen, E. Lin, Y. Liu. A novel Volume-Compensated Particle method for 2D elasticity and plasticity analysis. International Journal of Solids and Structures. 51 (9) (2014), pp. 1819-1833.

[24] K. E. Atkinson. An Introduction to Numerical Analysis, 2nd ed. New York: Wiley. (1989). 693 p.

[25] E. Barbieri, N. M. Pugno. A computational model for large deformations of composites with a 2D soft matrix and 1D anticracks. International Journal of Solids and Structures. 77 (2015), pp. 1-14.

[26] M. Endo, K. Takeuchi, T. Hiraoka, T. Furuta, T. Kasai, X. Sun, C.-H. Kiang, M. Dresselhaus. Stacking nature of graphene layers in carbon nanotubes and nanofibres. Journal of Physics and Chemistry of Solids. 58 (11) (1997), pp. 17071712.

[27] L. Jin, C. Bower, O. Zhou. Alignment of carbon nanotubes in a polymer matrix by mechanical stretching. Applied Physics Letters. 73 (9) (1998), pp. 1197-1199.

[28] A. Cao, C. Xu, J. Liang, D. Wu, B. Wei. X-ray diffraction characterization on the alignment degree of carbon nanotubes. Chemical Physics Letters. 344 (1) (2001), pp. 13-17.

[29] F. H. Gojny, M. Wichmann, U. Köpke, B. Fiedler, K. Schulte. Carbon nanotubereinforced epoxy-composites: enhanced stiffness and fracture toughness at low nanotube content. Composites Science and Technology. 64 (15) (2004), pp. 23632371.

[30] B.-K. Zhu, S.-H. Xie, Z.-K. Xu, Y.-Y. Xu. Preparation and properties of the polyimide/multi-walled carbon nanotubes (MWNTs) nanocomposites. Composites Science and Technology. 66 (3) (2006), pp. 548-554. 


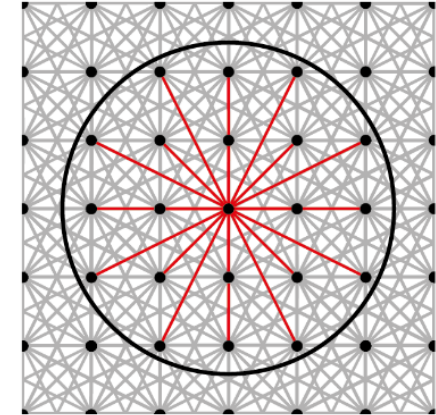

(a)

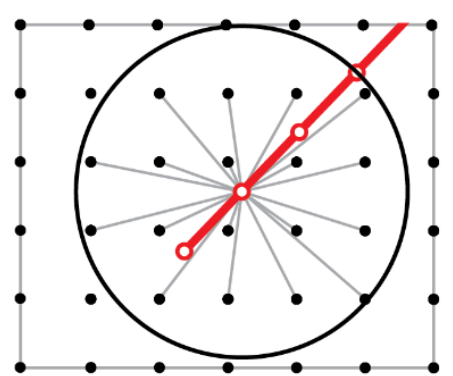

(c)

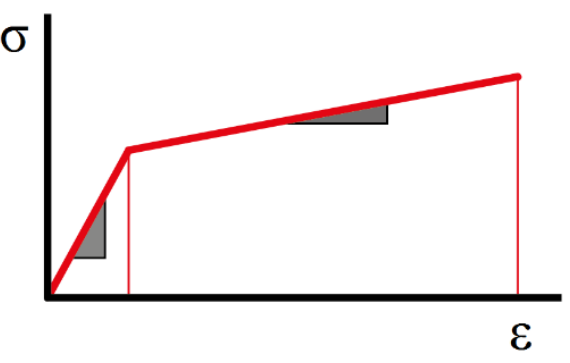

(b)

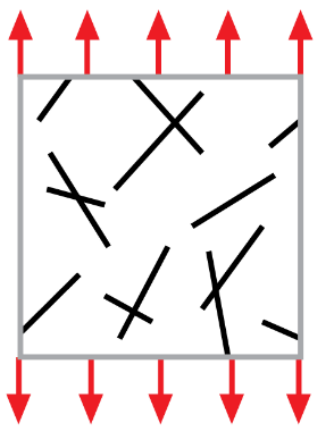

(d)

Fig. 1. (a) Non-local bonding between matrix nodes, where the circle represents an effective radius of interaction; (b) the assumed stress-strain behavior of matrix bonds; (c) non-local bonding between rigid line inclusion node and matrix nodes; and (d) schematic of the tensile test of the representative composite sub-domain. 


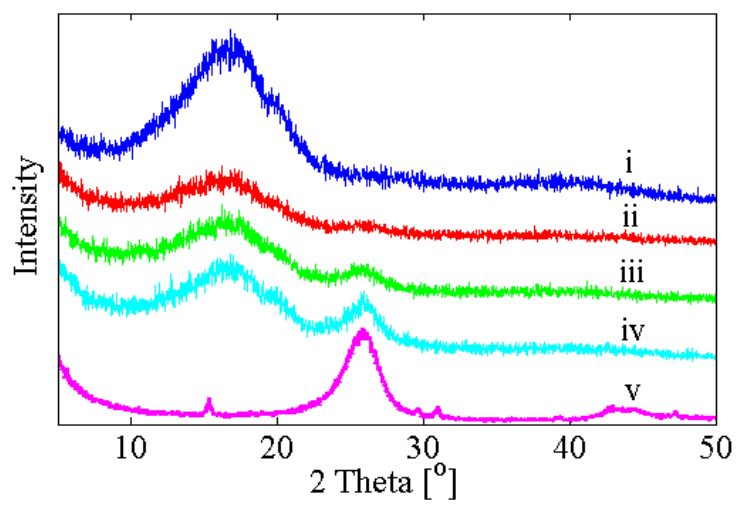

(a)

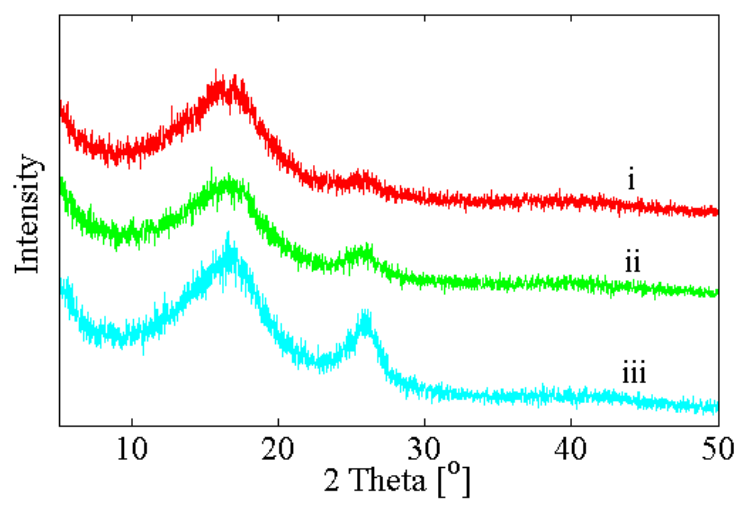

(b)

Fig. 2. XRD results for MWNT-latex thin films: (a) 0, 1, 2, and 3 wt $\%$ MWNT nonannealed films correspond to i-iv, respectively; $\mathrm{v}$ corresponds to pristine MWNTs; and (b) 1,2, and 3 wt $\%$ MWNT annealed films correspond to i-iii, respectively. 

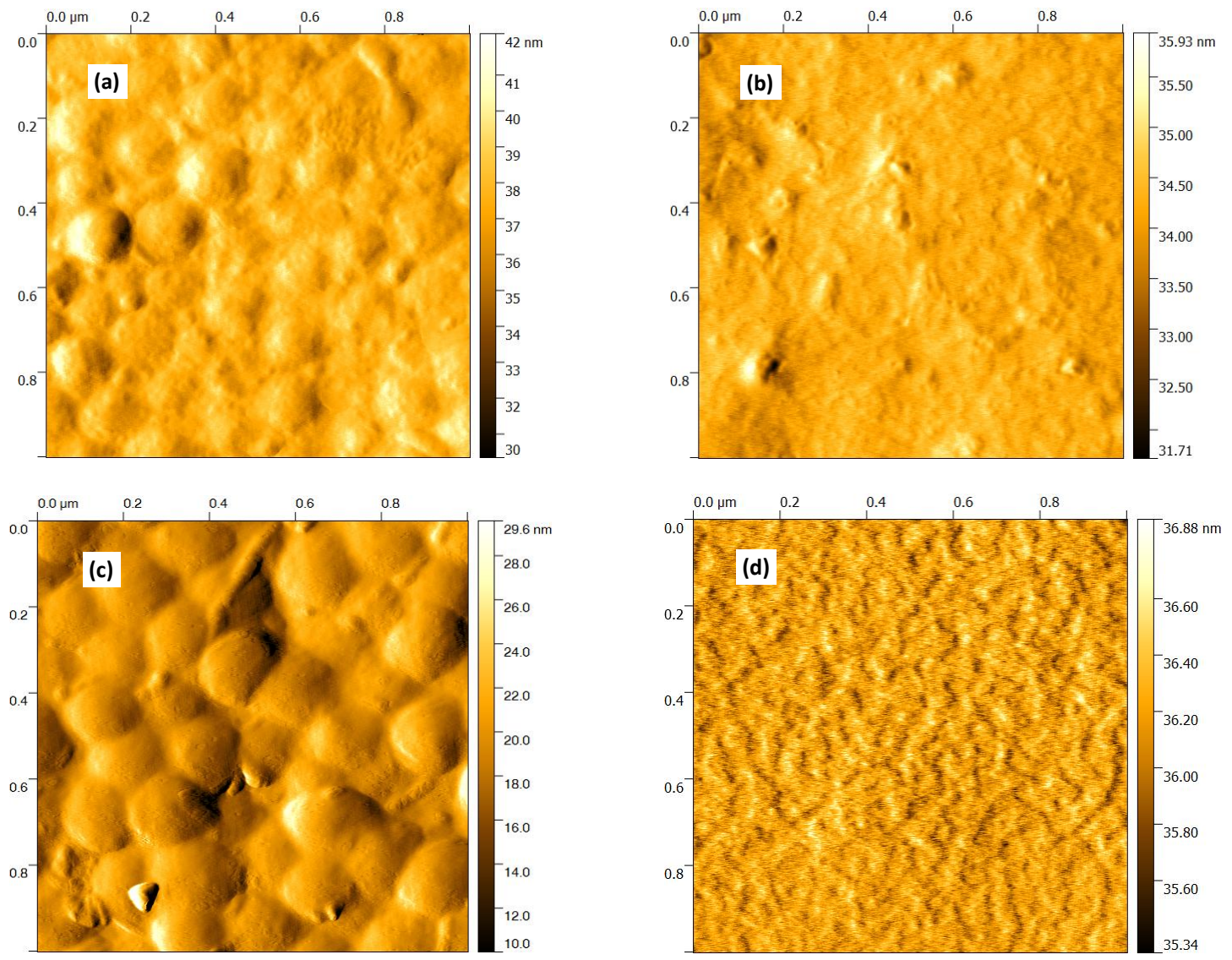

Fig. 3. AFM amplitude images of the surfaces of (a) non-annealed $0 \mathrm{wt} \%$, (b) annealed 0 wt $\%$, (c) non-annealed $1 \mathrm{wt} \%$, and (d) annealed $1 \mathrm{wt} \%$ MWNT-latex thin films. 

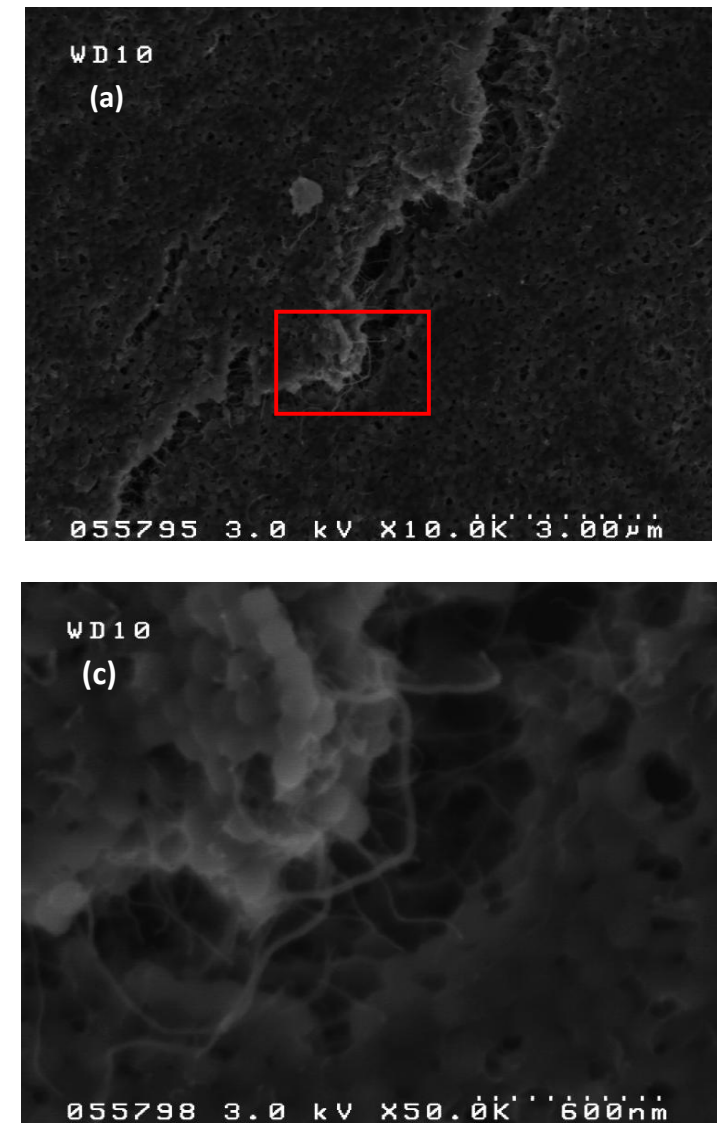
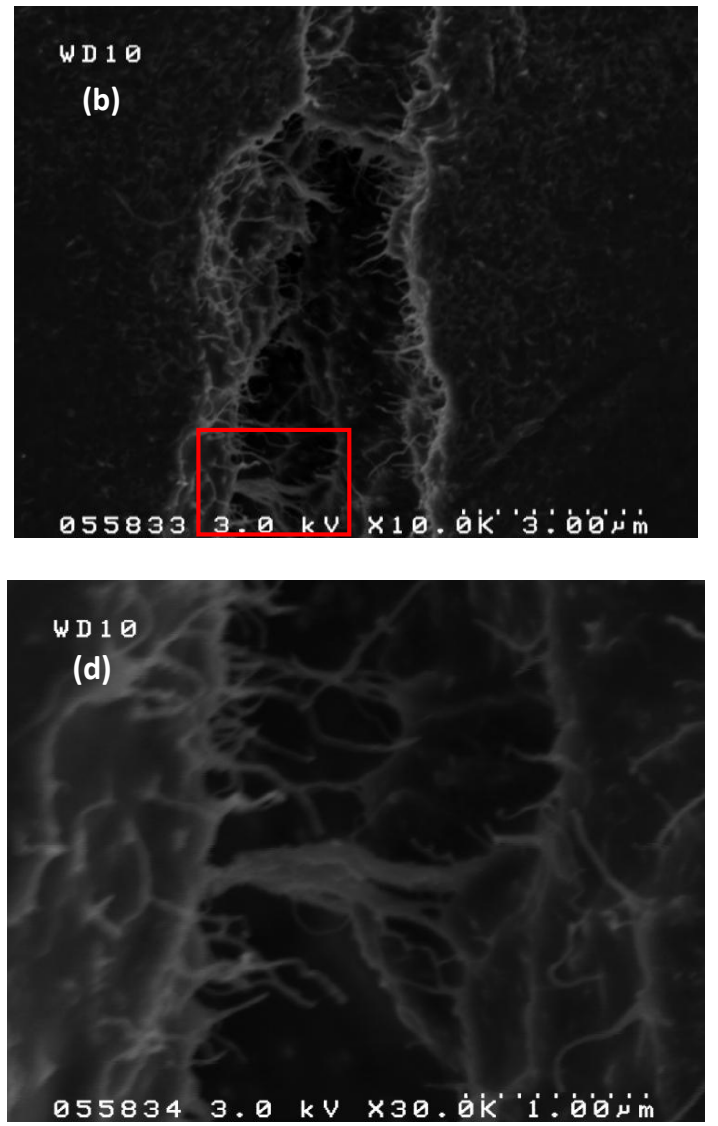

Fig. 4. SEM images of the fracture surfaces of (a) non-annealed and (b) annealed $1 \mathrm{wt} \%$ MWNT-latex thin films; (c) and (d) show higher magnifications of the areas marked by squares in (a) and (b), respectively. 


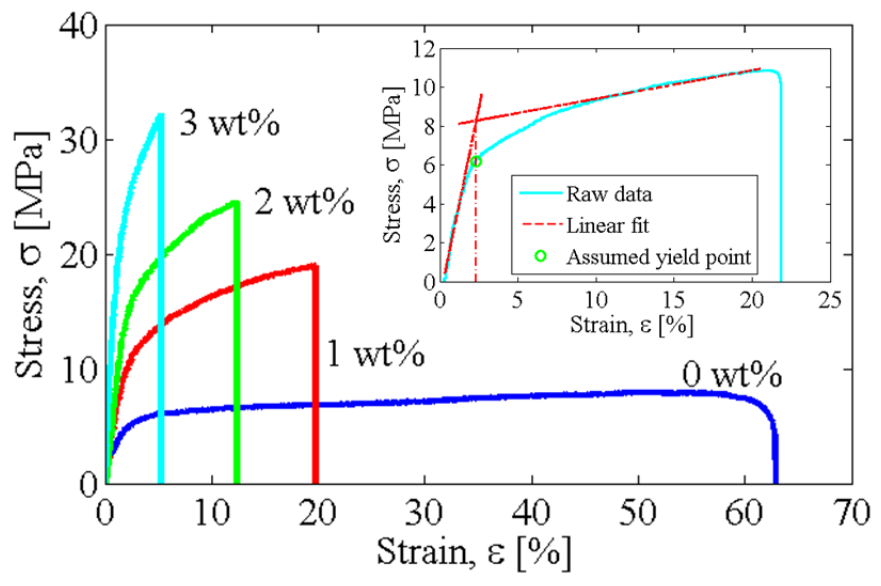

Fig. 5. Representative stress-strain curves of non-annealed 0, 1, 2, and $3 \mathrm{wt} \%$ MWNT-latex thin films; the inset shows the technique employed for determining the yield point. 


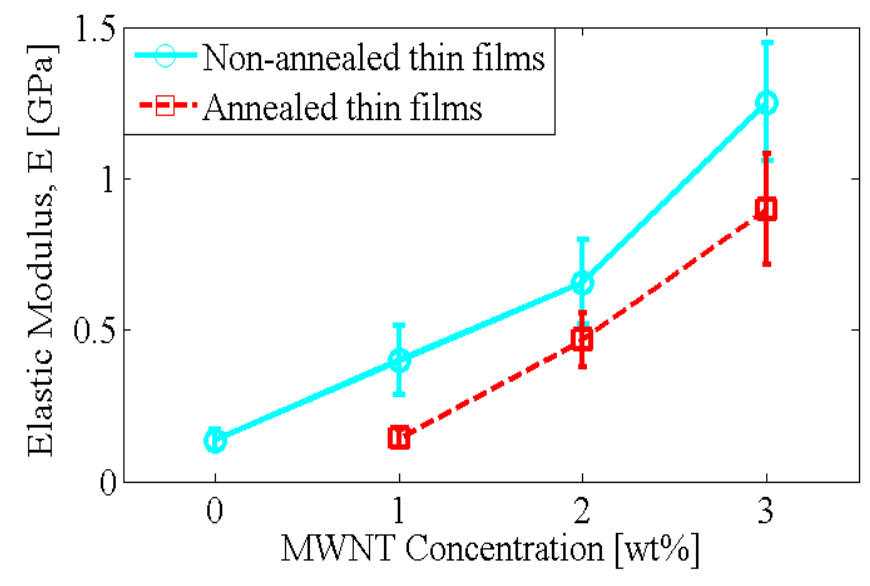

Fig. 6. The average elastic moduli (with standard deviations plotted as error bars) of nonannealed $0,1,2$, and $3 \mathrm{wt} \%$ and annealed 1, 2, and $3 \mathrm{wt} \%$ MWNT-latex thin films. 


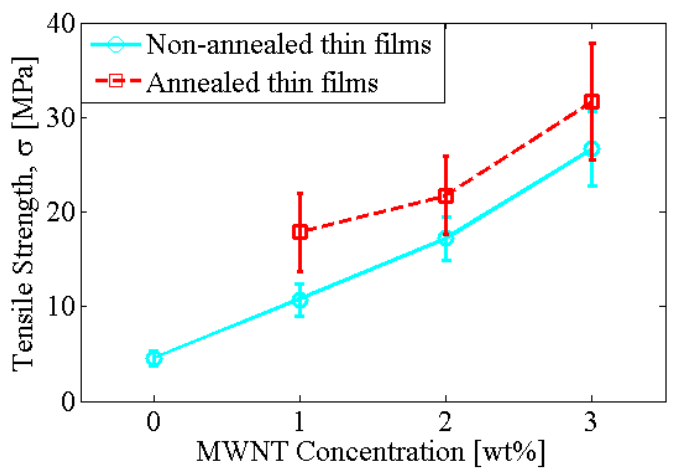

(a)

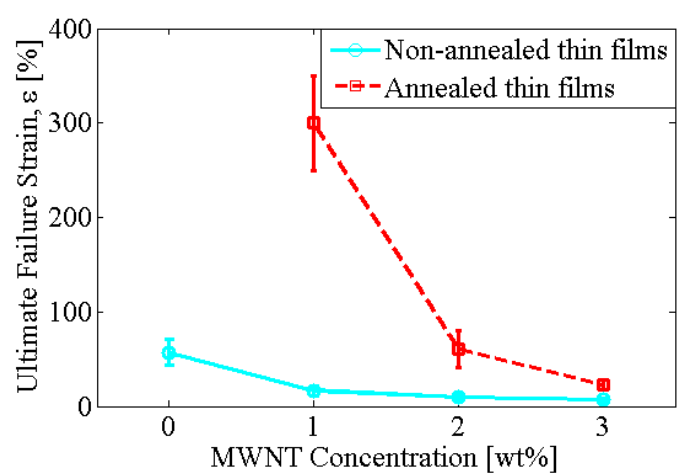

(b)

Fig. 7. The average (a) tensile strengths and (b) ultimate failure strains (with standard deviations plotted as error bars) of different non-annealed and annealed MWNT-latex film sample sets. 


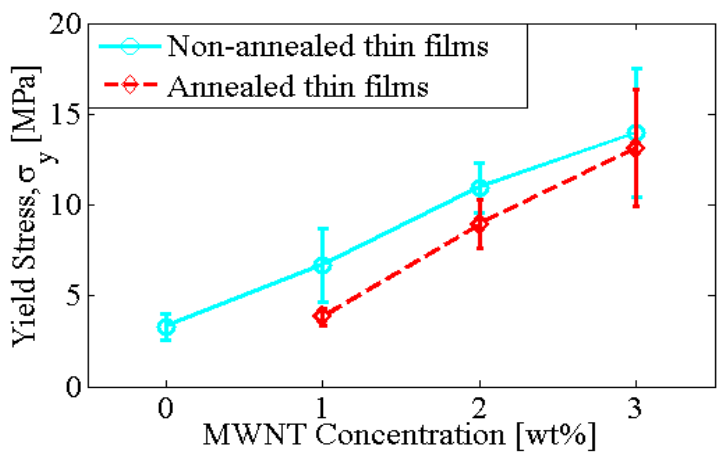

(a)

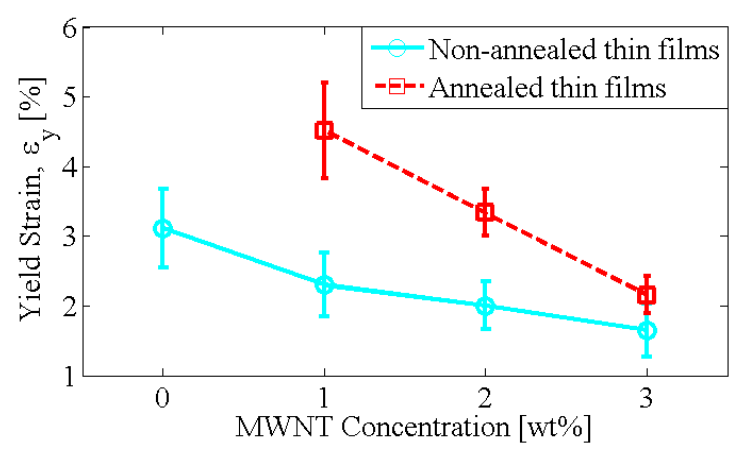

(b)

Fig. 8. The average (a) yield strength and (b) yield strains (with standard deviations plotted as error bars) of both non-annealed and annealed MWNT-latex thin film sample sets. 


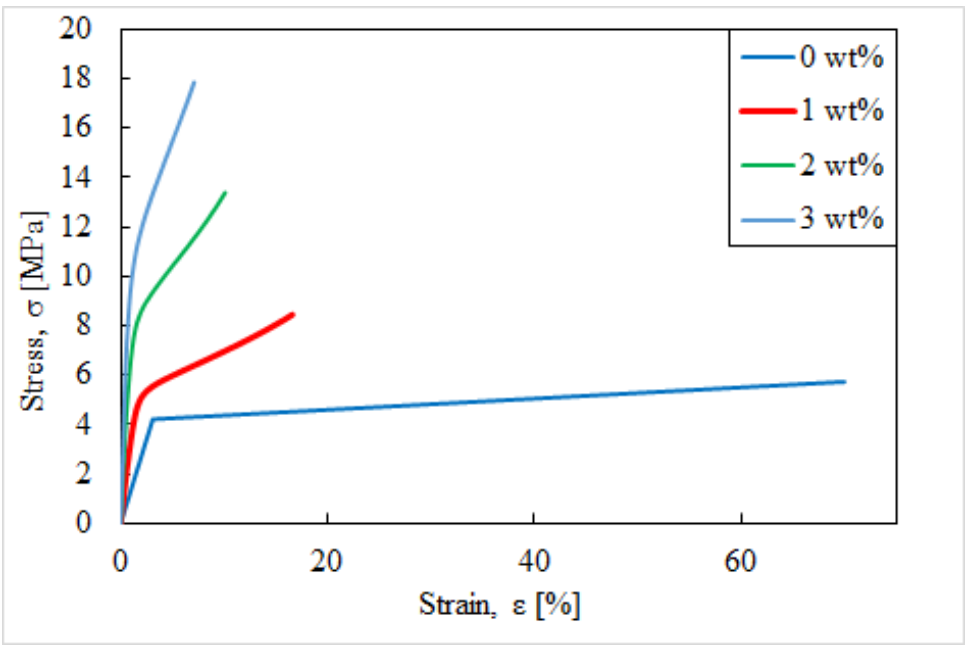

Fig. 9. Simulated stress-strain behavior for MWNT-latex thin films with different MWNT concentrations are shown. 
(a)

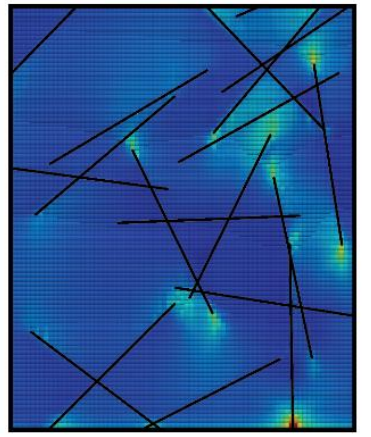

(b)

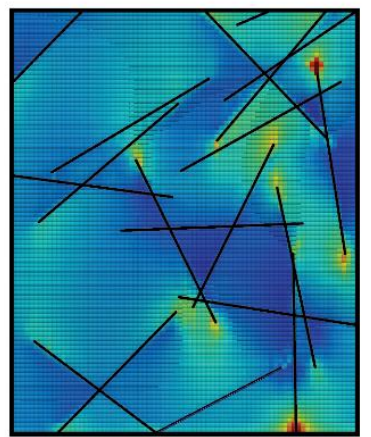

(c)

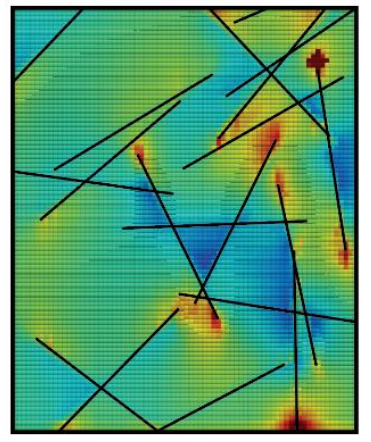

$\sigma[\mathrm{MPa}]$

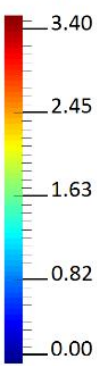

$\sigma[\mathrm{MPa}]$

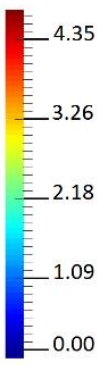

$\sigma[\mathrm{MPa}]$

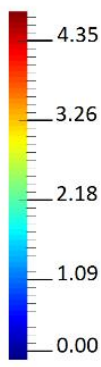

Fig. 10. Evolution of the distribution of stresses in a representative material portion for increasingly applied strains of (a) $1 \%$, (b) $2 \%$, and (c) $3 \%$ are presented. Line inclusions represent randomly distributed MWNTs. 


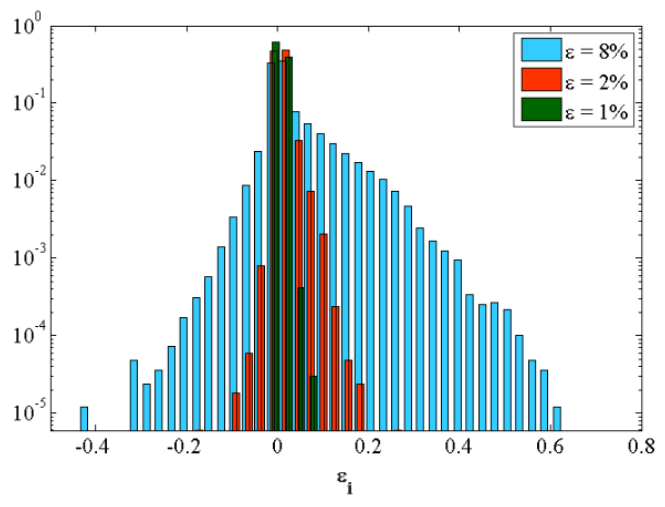

(a)

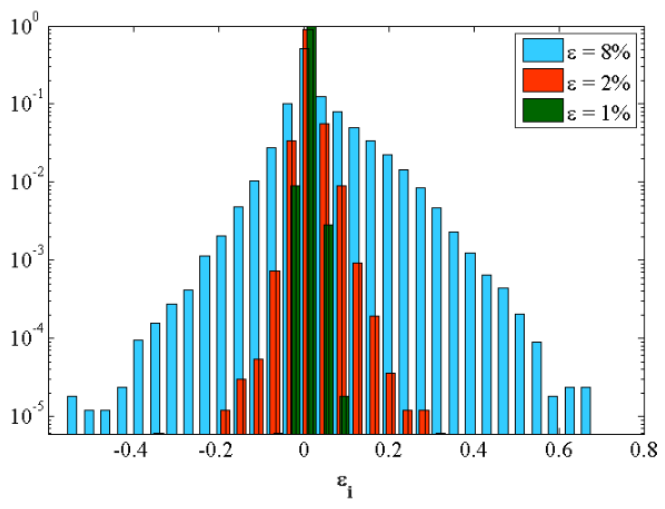

(b)

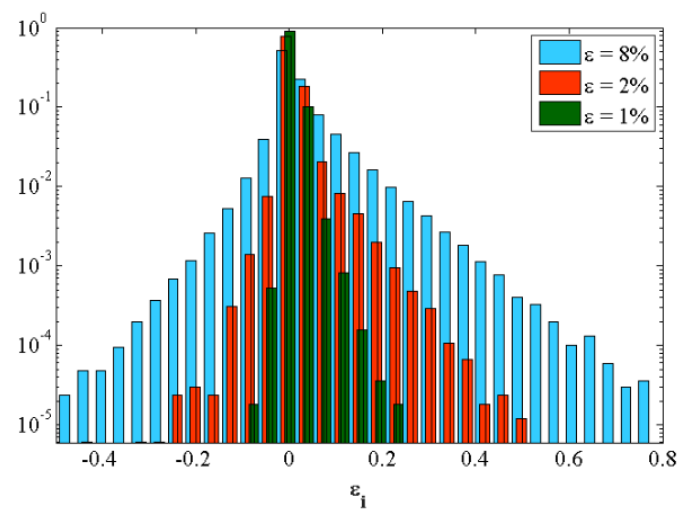

(c)

Fig. 11. Statistical distributions, $p\left(\varepsilon_{i}\right)$, of calculated strains, $\varepsilon_{i}$, in MWNT-latex films with (a) 1, (b) 2, and (c) $3 \mathrm{wt} \%$ MWNTs are shown. For each plot, distributions at applied external strains of $\varepsilon=1 \%, 2 \%$, and $8 \%$ are presented. 